\title{
Early peanut introduction wins over the HLA-DQA1*01:02 allele in the interplay between environment and genetics
}

\author{
Monali Manohar, Kari Christine Nadeau, and Maya Kasowski \\ Sean N. Parker Center for Allergy and Asthma Research, Department of Medicine, Stanford University School of Medicine, Stanford, California, USA
}

\begin{abstract}
The rising incidence of food allergy in children underscores the importance of environmental exposures; however, genetic factors play a major role. How the environment and genetics interact to cause food allergy remains unclear. The landmark Learning Early About Peanut Allergy (LEAP) clinical trial established that early peanut introduction protects high-risk infants, consistent with the tolerizing effects of gut exposure. In this issue of the JCI, Kanchan et al. leveraged the LEAP trial data to examine molecular genetic mechanisms of early sensitization. A previously identified HLA risk allele for peanut allergy (DQA1*01:02) was associated with peanut-specific IgG4 levels in consumers. Notably, IgC4 antibodies likely provide protection by reducing the binding of allergen to IgE. The association of the same allele with peanut allergy in avoiders while potentially conferring protection in consumers reinforces the need to integrate genetic information toward a personalized therapeutic strategy for the best outcome in addressing food allergies.
\end{abstract}

\section{Contextual associations between HLA alleles and peanut allergy}

The genetic factors that predispose an individual to atopy and food allergy including peanut have been evaluated through genome-wide association studies (GWAS) and candidate gene association studies (CGAS). Both approaches reveal a robust association between food allergy and HLA class II region (1), although how the interplay of genetic factors and environmental exposure, including early peanut introduction, modifies disease status had not been previously studied. In this issue of the JCI, Kanchan et al. (2) address this key gap through integrative analysis of multimodal data collected during the Learning Early About Peanut Allergy (LEAP) trial, a controlled peanut exposure study (3).

Kanchan et al. (2) discovered an association between peanut-specific Ara h 2specific IgG4 and the HLA-DQA1 ${ }^{*} 01: 02$ allele among participants in the consumption arm, who were exposed to sustained oral peanut protein. This association was further validated through the observed sequential-epitope-specific IgG4 expan-

Delated Article: https://doi.org/10.1172/JC1152070

Authorship note: KCN and MK are co-senior authors.

Conflict of interest: KCN reports stock options from IgGenix, Seed Health, ClostraBio, and ImmuneID, is director of the World Allergy Organization (WAO), an advisor at Cour Pharma, a consultant for Excellergy, Red Tree Ventures, Eli Lilly, and Phylaxis, a cofounder of Before Brands, Alladapt, Latitude, and IgGenix, and a National Scientific Committee member at the Immune Tolerance Network (ITN). KCN's patents include "Mixed allergen composition and methods for using the same" $(10 / 064,936)$, "Granulocyte-based methods for detecting and monitoring immune system disorders" (9891213), "Methods and assays for detecting and quantifying pure subpopulations of white blood cells in immune system disorders" (10114012), and "Methods of isolating allergen-specific antibodies from humans and uses thereof" (pending, application number PCT/US2019/032951).

Copyright: ( 2022 , Manohar et al. This is an open access article published under the terms of the Creative Commons Attribution 4.0 International License.

Reference information: J Clin Invest. 2022;132(1):e155609. https://doi.org/10.1172/JCl155609.

sion in $\mathrm{HLA}^{-D Q A} 1^{*} 01: 02$ carriers, but not noncarriers. Higher allergen-specific IgG4 has been linked with natural tolerance to a given allergen and is a biomarker of sustained unresponsiveness induced through allergen-specific immunotherapy $(4,5)$. Interestingly, the very same allele - HLADQA1 ${ }^{*}$ 01:02 - was associated with peanut allergy risk in the avoidance arm of the study. These findings indicate that peanut epitope presentation to $\mathrm{T}$ helper cells by HLA-DQ may shape the host immune response to favor peanut tolerance versus allergy depending on modifiable environmental factors such as early peanut introduction (Figure 1).

\section{Roadmap for possible future HLA-related investigations}

Future work may focus on more comprehensive genotyping of the HLA locus. It is important to consider, though, that all the HLA alleles in the host present various epitopes in concert, and studying the full complement of HLA allelic variants would best determine the cumulative impact of such presentation. Alternative pipelines affording parallel, comprehensive analysis of HLA-DR, -DP, and -DQ alleles are needed to effectively attribute immune response to the HLA molecule.

Kanchan and authors did not observe an association between HLA alleles and circulating (plasma) IL-4 and IL-10 levels in the consumption-arm participants (2). However, such evaluation would be more relevant had IL-4, IL-9, and IL-10 been induced through ex vivo peanut stimulation of participants' PBMCs, thus representing cytokines secreted by peanutreactive immune cells rather than measuring circulating levels.

Kanchan et al. invoked a dual-allergen exposure hypothesis, which posed that primary allergen exposure through skin is sensitizing and through the gut is tolerizing $(2,6)$. Thus, it would be worth comparing 


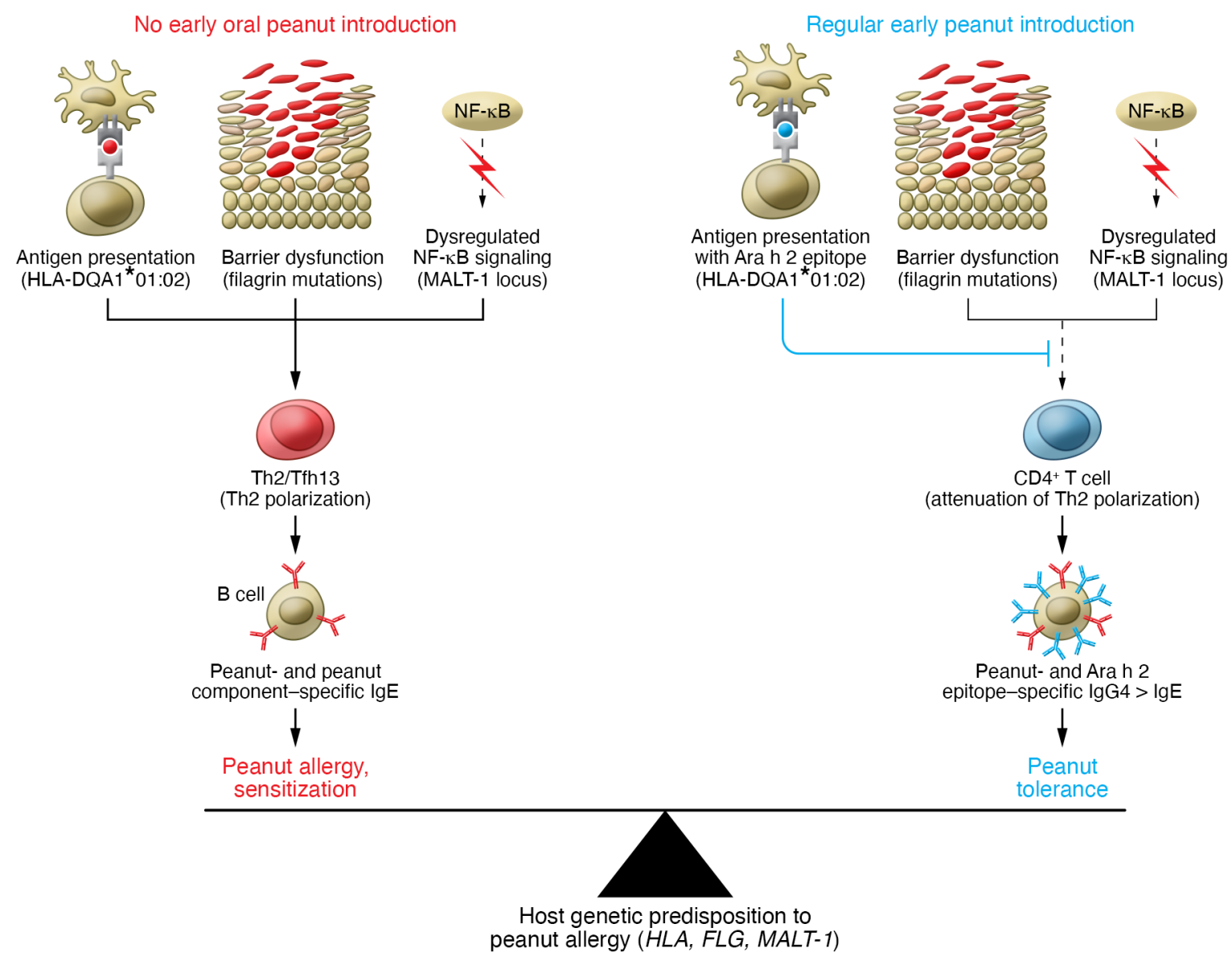

Figure 1. Tipping the balance in favor of peanut tolerance in the face of risk alleles. Genetic alleles, such as HLA-DQA*01:02, MALT-1, and FLG, predispose the carrier to the risk of developing peanut allergy. In the absence of oral peanut introduction in early infanthood (left panel), antigen presentation by HLA-DQ $A^{*} 01: 02$, barrier dysfunction caused by LFG mutations, and dysregulated NF-KB signaling due to MALT1 SNP together may promote polarization of naive CD4+ $T$ cells to Th2 and Tfh13 cells. Th2 and Thfh13 secrete type 2 cytokines, including IL-4, IL-5, and IL-13, which induce IgE class switching in B cells. Peanut and peanut component-specific IgE thus generated leads to peanut allergy pathogenesis. With early oral peanut introduction (right panel), peanut-derived epitopes, specifically Ara h 2 epitopes presented in the context of HLA-DQA*01:02, likely counter Th2 polarization of naive CD4+ $T$ cells. Attenuation of Th2 phenotype favors peanut epitope-specific lgG4 production over lgE production and could dampen the atopy-provoking effects of other risk alleles, thus leading to peanut tolerance.

Ara h 2 epitope presentation via skinresident and gastrointestinal-tract-resident antigen-presenting cells from HLADQA $1^{\star} 01: 02$ carriers using ex vivo assays.

\section{MALT1 is a risk allele with peanut epitope-independent effects}

This research team previously associated the MALT1 SNP rs57265082, a paracaspase-encoding gene, with peanut allergy in the avoidance arm of LEAP. They also found MALT1 to strongly associate with peanut-specific IgE as well as Ara h 1-, 2-, and 3-specific IgE, primarily in the avoidance group (7). In Kanchan et al. (2), the researchers observed MALT1 to have no primary effect on peanut-, Ara h 2-, or other peanut component-specific IgG4. Also, the association of MALT1 with peanutspecific IgE could not be attributed to certain sequential-epitope-specific expansion. Thus, the role of MALT1 allele in peanut allergy pathogenesis is likely independent of allergen-epitope presentation. The paracaspase encoded by MALT1 is a critical part of the CARMA1/BCL10/MALT1 complex, responsible for effective downstream activation of NF- $\kappa \mathrm{B}$ in $\mathrm{B}$ and $\mathrm{T}$ cells in response to receptor-mediated stimulation. Hence, an intuitive mechanistic role of SNP rs57265082 MALT1 is to promote IgE class switching on a broader scale through dysregulated NF- $\mathrm{NB}$ signaling. Nevertheless, deeper investigation for better understanding the role of MALT1 signaling in peanut allergy is warranted. Also, MALT1 has not been identified as a risk allele in peanut allergy in general population GWAS (7). Thus, cross-validation studies in an independent cohort are needed.

The outcome for participants who carry risk alleles, including FLG, DQ and MALT1, in the consumption arm after 12 months of avoidance (i.e., at week 72) in the context of LEAP-ON trial (8) is of interest too.

The results of Kanchan et al. (2) demonstrate the subtle, context-dependent effects of genetic risk factors. The alternate roles of $\mathrm{HLA}^{-\mathrm{DQA} 1{ }^{*} 01: 02}$ in the risk for peanut allergy and a favorable molecular response (Ara h 2 IgG4 levels) converge on a common mechanism of enhanced immune recognition. Whereas MALT1 associates with the immune response to many peanut components, HLA-DQA1 ${ }^{*} 01: 02$ is specific for Ara h 2, 
consistent with a narrower role (Figure 1). An important direction for future work involves exploring the generalizability of the LEAP story to other allergens. The dual-allergen exposure hypothesis is thought to apply broadly to food allergies. Are there other HLA allele-antigen dyads that convey risk in the absence of early consumption? Such data provide an understanding of the fundamentals of allergic sensitization and may also have practical clinical value for triaging at-risk individuals for oral immunotherapy (OIT) response that would confer lifelong benefit.

\section{Importance of GWAS in the context of therapeutic interventions}

Kanchan et al. (2) also highlight the value of studying genetic effects in clinical trial cohorts. Although the scale is modest compared with that of GWAS, the LEAP trial continues to provide complementary and unique insights into the genetics of peanut allergy. A generalizable lesson from the LEAP experience may include the value of studying gene-environment interactions during critical developmental windows of exposure. Although HLA-DQA1 ${ }^{\star} 01: 02$ was previously identified by GWAS as a risk locus for peanut allergy, the link to peanut-specific IgG4 was uncovered by studying the response of high-risk infants to a potential therapeutic intervention. The clinical trial setting not only enabled the controlled perturbation of specific study populations, but also involved rich longitudinal phenotyping, including disease-relevant molecular studies (e.g., allergen-specific immunoglobulin lev- els). Enrolling children in clinical trials was essential to studying early etiologic events. For future investigations, it will also be important to recruit individuals of non-European-derived genetic ancestries in order to understand population differences in allergy, prioritize causal variants, and address iniquities in the allocation of GWAS resources. One can imagine replicating the success of the LEAP trial to study the impact of other developmentally timed exposures that may moderate the effects of genetic susceptibility (e.g., microbial exposures). Studying high-risk children has the benefit of revealing therapeutic strategies for those in greatest need. In addition, the large effect sizes (as observed in the LEAP cohort) may also help identify informative contexts for large-scale, general population analyses.

As the authors point out, consumption is so effective in preventing peanut allergy that it is not possible from their data to assess whether HLA-DQA1 ${ }^{*} 01: 02$ associates with tolerance in consumers. Thus, the clinical relevance of this gene-environment interaction awaits future work. Despite the limitations, Kanchan et al. (2) undoubtedly highlight how a therapeutic intervention such as peanut consumption through OIT may differentially affect participants with varying genotypes. Although it remains to be tested, past and current LEAP analyses raise the intriguing possibility that early childhood genotyping may help prioritize at-risk individuals for OIT.

\section{Acknowledgments}

This work was supported by The Sean N. Parker Center for Allergy and Asthma
Research at Stanford University, NIAID grants R01AI140134 and U19AI104209, and ITN funding. MK is supported by a KL2 Mentored Career Development Award KL2TR003143 and an NIH Director's New Innovator Award DP2HG012480. We would like to thank Vanitha Sampath for her help in preparation of this Commentary.

Address correspondence to: Kari C. Nadeau, Sean N. Parker Center for Allergy and Asthma Research at Stanford University, Division of Pulmonary, Allergy \& Critical Care Medicine, Department of Medicine, Stanford University School of Medicine, 240 Pasteur Dr., BMI Rm.1755, Palo Alto, California 94304, USA. Phone: 650.867.4592; Email:knadeau@stanford.edu.

1. Kanchan K, et al. Current insights into the genetics of food allergy. J Allergy Clin Immunol. 2021;147(1):15-28.

2. Kanchan K, et al. HLA alleles and sustained peanut consumption promote IgG4 responses in subjects protected from peanut allergy. J Clin Invest. 2021;132(1): 152070.

3. Du Toit G, et al. Randomized trial of peanut consumption in infants at risk for peanut allergy. NEngl J Med. 2015;372(9):803-813.

4. Tordesillas L, Berin MC. Mechanisms of oral tolerance. Clin Rev Allergy Immunol. 2018;55(2):107-117.

5. Chinthrajah RS, et al. Sustained outcomes in oral immunotherapy for peanut allergy (POISED study): a large, randomised, double-blind, placebo-controlled, phase 2 study. Lancet. 2019;394(10207):1437-1449.

6. Du Toit G, et al. Prevention of food allergy. J Allergy Clin Immunol. 2016;137(4):998-1010.

7. Winters A, et al. The MALT1 locus and peanut avoidance in the risk for peanut allergy. JAllergy Clin Immunol. 2019;143(6):2326-2329.

8. Du Toit G, et al. Effect of avoidance on peanut allergy after early peanut consumption. $N$ EnglJ Med. 2016;374(15):1435-1443. 